\title{
Feasibility of Oil Slick Detection Using BeiDou-R Coastal Simulation
}

\author{
Yun Zhang, Shanshan Chen, Zhonghua Hong, Yanling Han, \\ Binbin Li, Shuhu Yang, and Jing Wang \\ College of Information Technology, Shanghai Ocean University, 999 Hu-Chenghuan Road, Shanghai 201306, China
}

Correspondence should be addressed to Zhonghua Hong; zhhong@shou.edu.cn

Received 28 October 2016; Accepted 5 January 2017; Published 1 February 2017

Academic Editor: Hajime Seya

Copyright (c) 2017 Yun Zhang et al. This is an open access article distributed under the Creative Commons Attribution License, which permits unrestricted use, distribution, and reproduction in any medium, provided the original work is properly cited.

Oil spills, which can cause severe immediate and long-term harm to marine ecological environments for decades after the initial accident, require rapid and accurate monitoring. Currently, optical and radar satellite images are used to monitor oil spills; however, remote sensing generally needs a long revisit period. Global Navigation Satellite System reflected signals (GNSS-R) can provide all-weather and all-day ocean monitoring and is therefore more suitable for oil spill monitoring. To assess the feasibility of the BeiDou Navigation Satellite System reflected signals (BeiDou-R) in detecting oil slicks, a BeiDou-R coastal simulated experiment is performed in this study on the oil slick distribution of an oil pipeline explosion accident. We set up an observation point and selected observation satellites, and a delay-Doppler map (DDM) of an oil-slicked sea surface under coastal scenarios was created by combining the mean-square slope (MSS) model for oil-slicked/clean surfaces and the Zavorotny-Voronovich (Z-V) scattering model. DDM simulation of the coastal scenarios effectively represents the scattering coefficient distribution of the presence of an oil slick. Theoretical analysis revealed that oil slicks can be detected within a radius of less than $5 \mathrm{~km}$ around the specular reflection point (SP) for BeiDou-R coastal simulation.

\section{Introduction}

Pollution by oil slicks in open ocean areas and coastal waters, whether accidental or deliberate, is a serious threat to marine environments, the economy, and human life. An oil spill occurs somewhere in the world almost every day of the year. The Deepwater Horizon oil spill accident in the Gulf of Mexico, which began on April 20, 2010, directly affected $176,000 \mathrm{~km}^{2}$ of the Gulf's surface [1]. In addition, an oil pipeline explosion occurring in the Yellow Sea on November 22,2013 , heavily contaminated more than $3000 \mathrm{~m}^{2}$ of the sea surface; the direct economic loss of this accident has been calculated as 750 million Yuan [2]. To reduce the impact of oil spill disasters on the environment and human health, accurate monitoring of oil spills at sea is of particularly importance. Operational oil spill monitoring is currently accomplished using a combination of optical and radar satellite images $[3,4]$. However, because optical satellites are easily affected by weather, they cannot detect oil slicks under adverse conditions. Radar is used for monitoring under all-weather conditions and all day; however, the observation model is often in side-scan mode, which significantly affects the accuracy of oil spill detection owing to speckle noise in Synthetic Aperture Radar (SAR) images. In contrast, Global Navigation Satellite System reflected signals (GNSS-R) provide reflectivity information of the L-band, which is capable of penetrating cloud cover. In addition, the most important difference between GNSS and traditional remote sensing applications is that GNSS remote sensing is a bistatic technique. The transmitter and the receiver are not collocated, resulting in the transmitted GNSS signal reflecting or scattering in a forward direction; traditional remote sensing applications use more conventional back-scattered signals [5]. Moreover, GNSS-R payloads installed on different platforms can receive signals from the navigation satellites in large parts of the ocean and can provide long-term monitoring of oil slicks in all-weather conditions and all day.

The powerful GNSS-R was originally put forward in 1993 by Martin-Neira [6] as a new technique for ocean altimetry. Since then, reflections of GNSS signals from different types of surfaces have been widely studied. Several remote sensing applications presently using GNSS-R technology include 


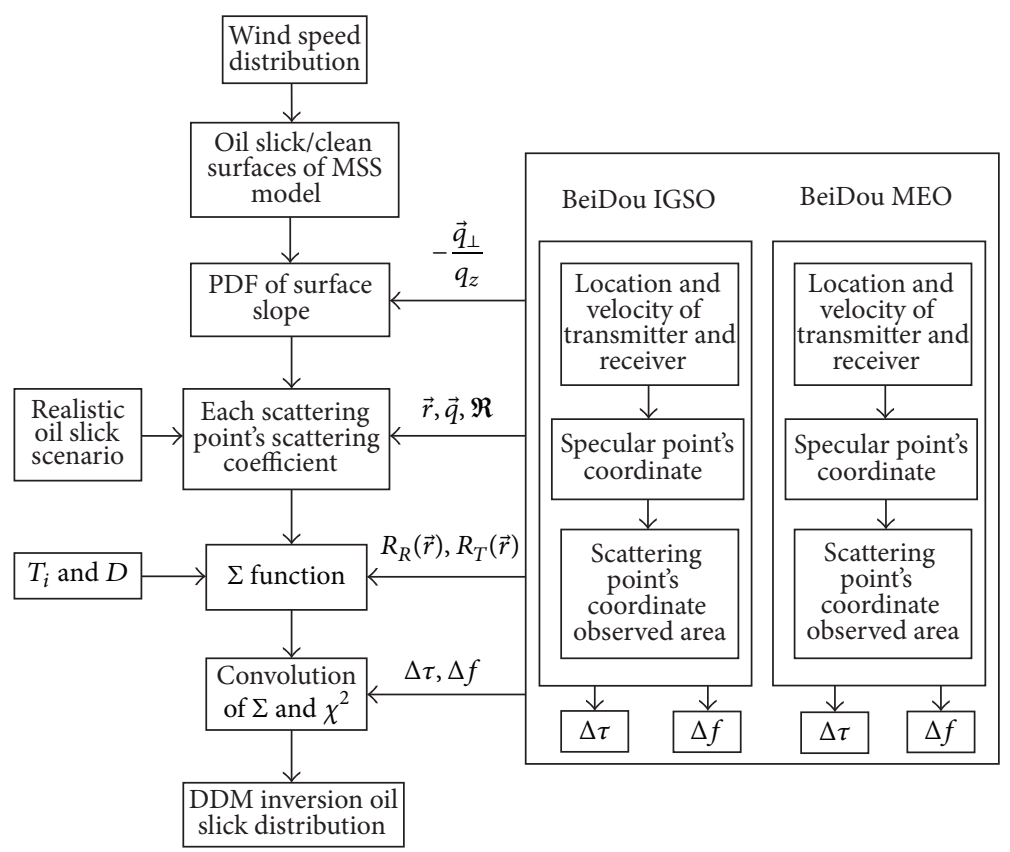

Figure 1: Flowchart of delay-Doppler map (DDM) simulation.

ocean altimetry [7-10], ocean wind [11-13], ocean wave sensing [14-16], and sea ice detection [17-19]. In addition, the possibility of detecting an oil-slicked sea surface using GNSS$\mathrm{R}$ technology simulation has been investigated by Valencia et al. $[20,21]$. This technique is based mainly on scattering coefficient retrieval using a delay-Doppler map (DDM). Li and Huang (2013) applied the methodology of a spatial integration approach (SIA) to sea surface oil slick detection simulation using DDM obtained under a simplified scenario with elevation angle of $90^{\circ}[22]$. This method has also been extended to general scenarios [23]; details of the DDM process simulation have been previously reported [24].

In addition to Global Positioning System (GPS), Global Navigation Satellite System (GNSS), and Galileo, other GNSS instruments currently in development include China's BeiDou constellation [25]. The development of the BeiDou system provides a new signal source to monitor ocean. Previous research focuses mainly on oil spill simulation detection using GPS reflected signals (GPS-R) technology. However, GPS uses only Medium Earth Orbit (MEO) satellites. Compared with existing GNSS constellations, the Chinese BeiDou satellite navigation system consists of MEO satellites in addition to Inclined Geosynchronous Orbit (IGSO) and Geostationary Earth Orbit (GEO) satellites. Until March 2015, five GEO, five IGSO, and six MEO BeiDou navigation satellites were in orbit. Moreover, the mixed constellation structure of BeiDou allows persistent monitoring of a given ocean area from multiple angles simultaneously. Currently, very few studies are available on ocean oil slick detection simulation using BeiDou-R. To test the performance of oil slick detection by using the BeiDou-R, the present study uses coastal simulation to detect oil-slicked ocean surfaces by using BeiDou IGSO and MEO satellites. Because BeiDou GEO satellites are almost stationary relative to Earth, they cannot use relative motion to produce Doppler frequency shift of DDM and are not considered in simulation experiments. The coastal simulation in this study is based on an actual oil spill accident that caused marine oil pollution. Based on the polluted area, we set up an observation point and select the corresponding observation satellites to monitor the oil spill conditions. Simulation DDM of the oil-slicked surface is then calculated by using the mean-square slope (MSS) model for oil-slicked/clean surfaces and the $\mathrm{Z}-\mathrm{V}$ scattering model.

\section{Simulation Approach}

2.1. Framework. In this paper, a realistic scenario is simulated to improve the reliability of BeiDou-R for detection oil spill, which is derived from QuickBird multispectral images. Furthermore, this paper gives comparative analysis on the performance difference between BeiDou IGSO and MEO satellites for oil slick detection.

Figure 1 shows the specific DDM simulation process for an oil slick scenario. In the proposed framework, coastal simulation experiments using two BeiDou IGSO and MEO satellites are conducted on the basis of a realistic oil slick scenario. First, according to the geographic location information of the oil spill area, we set up the coastal observation point and selected the corresponding observation satellites. The location of the specular reflection point (SP) and the scattering point needs to be implemented according to the design of the coastal scenario. Under the conditions of observing the scattering coefficient distribution of a realistic oil slick, DDM simulation of the oil-slicked surface was then calculated by combining the MSS model for oil-slicked/clean surfaces and the $\mathrm{Z}-\mathrm{V}$ scattering model. Finally, DDM simulation had been used to retrieve the scattering coefficient distribution of the presence of an oil slick based on the signal delay and 


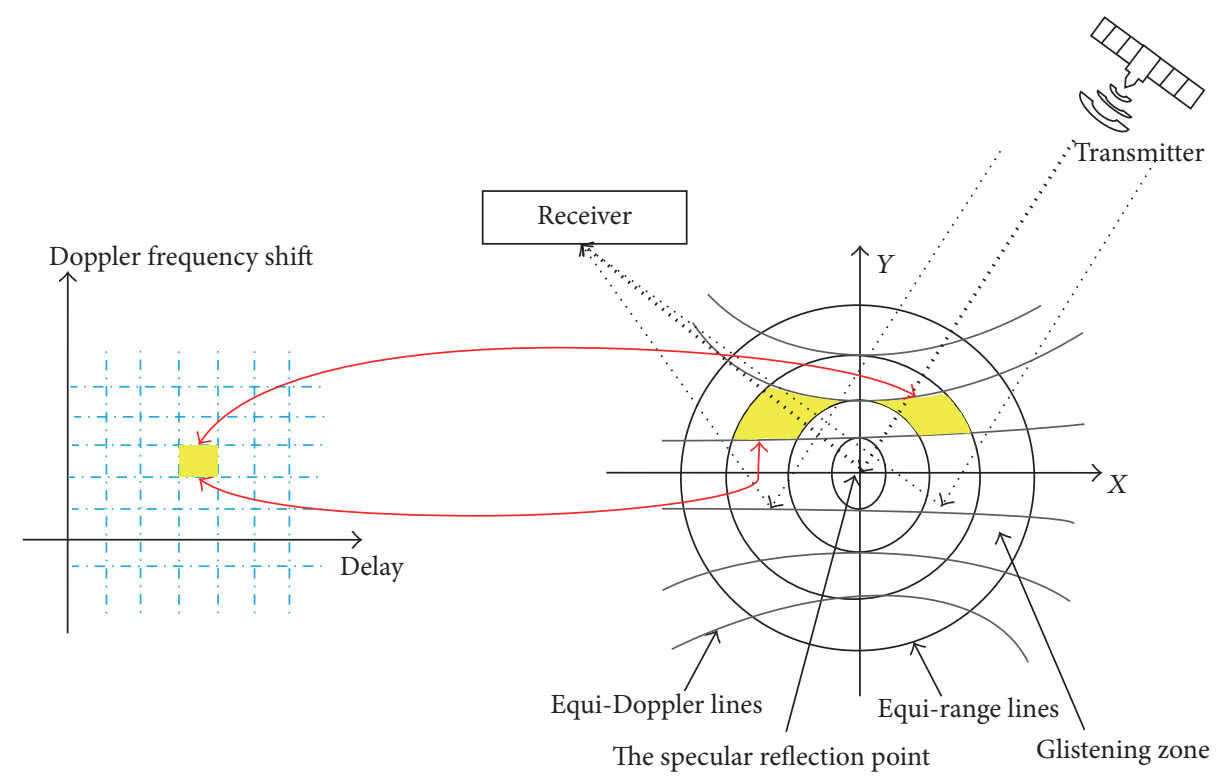

FIGURE 2: Corresponding relationship between the scattering units and the delay-Doppler units.

Doppler frequency shift. Oil slicks were detected within a radius coverage area around the SP based on the DDM. These methods are discussed in detail in the following sections.

2.2. DDM Theory. In an actual GNSS-R operational system, GNSS signals need to be received and processed using a bistatic configuration. GNSS-R receiver measurement of the sea-scattered signal is based on scattering from the different scattering units of the sea surface. The differences in delay and Doppler frequency shift correspond to the different scattering units of the sea surface, as shown in Figure 2.

GNSS signals scattered from the ocean are affected by the reflecting surface when it presents oil slicks. In such cases, the changes induced by the oil slick surface can be described by the correlation power waveform of the scattered signal in different delay and Doppler frequency shift values.

The DDM accounts for the correlation power distribution of the sea surface signals scattered as a function of delay and Doppler frequency shift with respect to the SP by using the GNSS-R scattering model proposed by Zavorotny and Voronovich expressed as [26, 27]

$$
\left\langle\left|Y\left(\Delta \tau, \Delta f_{D}\right)\right|^{2}\right\rangle=\Sigma\left(\Delta \tau, \Delta f_{D}\right) * \chi^{2}\left(\Delta \tau, \Delta f_{D}\right),
$$

where $*$ indicates two-dimensional convolution and $\chi$ is the ambiguity function described as

$$
\chi^{2}\left(\Delta \tau, \Delta f_{D}\right)=\Lambda^{2}(\Delta \tau) \cdot\left|S\left(\Delta f_{D}\right)\right|^{2},
$$

where the triangle of the $\Lambda(\Delta \tau)$ function is described as $\Lambda(\Delta \tau)=1-|\Delta \tau| / \tau_{c}$ if $|\Delta \tau|<\tau_{c}$ and $\Lambda(\Delta \tau)=0$ otherwise, $\tau_{c}$ is the length of a chip of the C/A code (BeiDou $\tau_{c}=1 \mathrm{~ms} /$ 2046, GPS $\left.\tau_{c}=1 \mathrm{~ms} / 1023\right)$, and $S\left(\Delta f_{D}\right)=\sin \left(\pi \Delta f_{D} T_{i}\right) /$ $\left(\pi \Delta f_{D} T_{i}\right) \times \exp \left(-\pi i \Delta f_{D} T_{i}\right)$.

In the application of the GNSS-R signals, the delay and the Doppler at the SP are used as the reference point. $\Delta \tau$ is the delay of the scattering point with respect to the SP expressed as

$$
\Delta \tau=\tau(\vec{r})-\tau(0)=\frac{\left(R_{T}(\vec{r})+R_{R}(\vec{r})\right) f}{c}-\tau(0),
$$

where $f$ is the frequency of C/A code (BeiDou $f=2.046 \times$ $10^{6} \mathrm{~Hz}$, GPS $\left.f=1.023 \times 10^{6} \mathrm{~Hz}\right), R_{T}(\vec{r})$ is the distance from the transmitter to the scattering point, $R_{R}(\vec{r})$ is the distance from the receiver to the scattering point, and $c$ is the speed of light. $\Delta f$ is the Doppler frequency shift of the scattering points with respect to the SP expressed as [28]

$$
\Delta f=f_{D}(\vec{r})-f_{D}(0)=\frac{\left(\vec{V}_{T} \cdot \widehat{m}-\vec{V}_{R} \cdot \widehat{n}\right)}{\lambda}-f_{D}(0),
$$

where $\vec{V}_{T}$ and $\vec{V}_{R}$ are the velocities of transmitter and receiver, respectively; $\widehat{m}$ is the unit vector of the incident wave; and $\hat{n}$ is the unit vector of the scattered wave. The Doppler frequency shift of the signal at different scattering points across the surface can be accurately determined by using (4) with respect to the known positions and velocities of the transmitter, receiver, and SP. Before calculating the received power, $\Sigma$ of each scattering point is determined as

$$
\Sigma\left(\Delta \tau, \Delta f_{D}\right)=\frac{T_{i}^{2} D^{2}(\vec{r}) \sigma^{0}(\vec{r})}{4 \pi R_{R}^{2}(\vec{r}) R_{T}^{2}(\vec{r})} d s,
$$

where $T_{i}$ is the coherent integration time, $D$ is the antenna radiation pattern, and $\vec{r}$ is the displacement vector of a scattering point from the SP. The SP can be calculated by using the known locations of the receiver and transmitter; details of the calculation are shown elsewhere [5]. The expression of each scattering point of the scattering coefficients over the sea surface is [26]

$$
\sigma^{0}(\vec{r})=\pi|\Re|^{2}\left(\frac{|\vec{q}|}{q_{z}}\right)^{4} P_{\mathrm{pdf}}\left(-\frac{\vec{q}_{\perp}}{q_{z}}\right),
$$


where $|\Re|^{2}$ is the Fresnel reflection coefficient; $\vec{q}$ is the scattering vector, which is defined as $\vec{q}=\kappa(\widehat{n}-\widehat{m})=\vec{q}_{\perp}+q_{z} \widehat{z}$; and $\kappa$ is the carrier wave number. $P_{\mathrm{pdf}}\left(-\vec{q}_{\perp} / q_{z}\right)$ represents the probability density function (PDF) of the given sea surface slope. Soulat presented the PDF of surface slopes for arbitrary wind direction [29]. Here, we assume that the wind direction is along the $x$-axis of the Earth-centered $X-Z$ incidence (ECXI) system. The slope PDF can be written as [26]

$$
\begin{aligned}
& P_{\mathrm{pdf}}\left(-\frac{\vec{q}_{\perp}}{q_{z}}\right)=\frac{1}{2 \pi \sqrt{\sigma_{\mu}^{2} \sigma_{c}^{2}}} \\
& \cdot \exp \left[-\frac{1}{2}\left(\frac{\left(-q_{\perp, c} / q_{z}\right)^{2}}{\sigma_{c}^{2}}+\frac{\left(-q_{\perp, \mu} / q_{z}\right)^{2}}{\sigma_{\mu}^{2}}\right)\right],
\end{aligned}
$$

where $-q_{\perp, c} / q_{z}$ and $-q_{\perp, \mu} / q_{z}$ represents the upwind and crosswind ocean slope, respectively; $\sigma_{c}^{2}$ is a crosswind MSS component; and $\sigma_{\mu}^{2}$ is an upwind MSS component. According to the Cox and Munk MSS model, the presence of oil slicks on the sea surface translates into changes in the surface MSS [30]. Accordingly, the relationship among the ocean surface's MSS and the wind speed (WS) can be formulated as

$$
\begin{aligned}
\sigma_{c, c}^{2} & =0.003+1.92 * 10^{-3} U_{10}, \\
\sigma_{\mu, c}^{2} & =3.16 * 10^{-3} U_{10} .
\end{aligned}
$$

To model the effect of the oil slick presence, the oil slick surface's MSS is computed by using the appropriate relationship with the WS as

$$
\begin{aligned}
& \sigma_{c, s}^{2}=0.003+0.84 * 10^{-3} U_{10}, \\
& \sigma_{\mu, s}^{2}=0.005+0.78 * 10^{-3} U_{10},
\end{aligned}
$$

where the second subscripts of MSS, that is, $c$ and $s$, represent clean and oil-slicked surfaces, respectively and $U_{10}$ is the WS at the height of $10 \mathrm{~m}$ from the sea surface. This MSS model was originally proposed for optical wavelengths signals. To apply the model to L-band GNSS-R signals, an empirical modification was proposed by Katzberg et al. [31] in 2006. In this paper, we adopted this empirical MSS model for the clean and slicked regions in the simulation.

Oil slicks on the sea surface cause changes in the scattering coefficient distribution over the observed surface and thus change the corresponding entire DDM. By using this oil slick model, the impacts of the oil slick on the DDM can be distinguished.

\section{Simulation Scenarios}

The coastal simulation experiment is based on the oil slick distribution from an oil pipeline explosion that occurred in the district of Qingdao, China. The oil spill distribution data were used as input for the DDM simulation by using QuickBird multispectral images. According to the location of oil spill on the sea surface, we assumed two observation

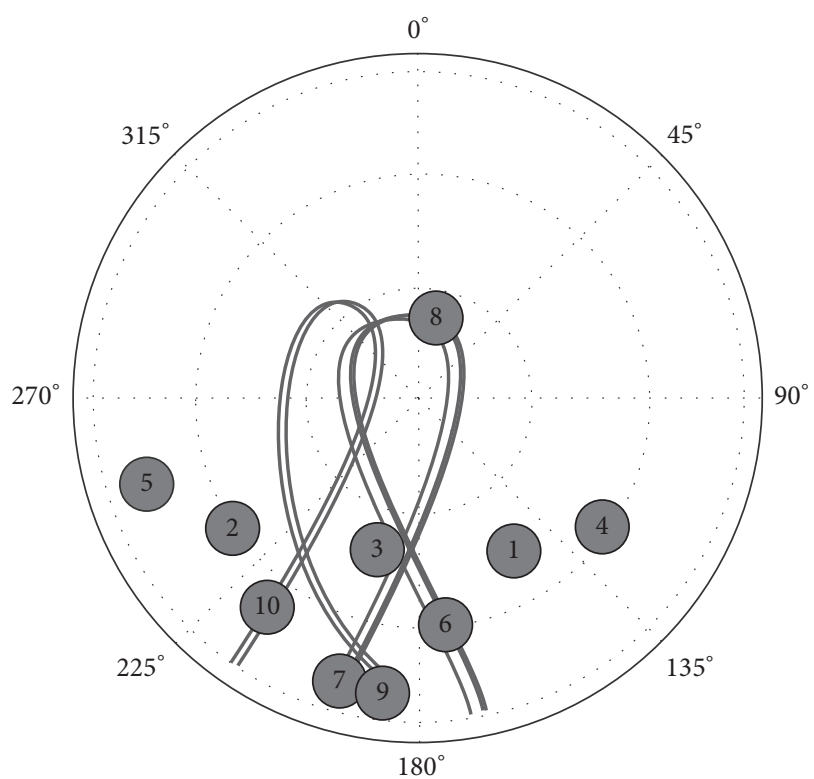

FIGURE 3: Skyplot point of Inclined Geosynchronous Orbit (IGSO) C06 for experiment I (08:10 LT, November 27, 2013).

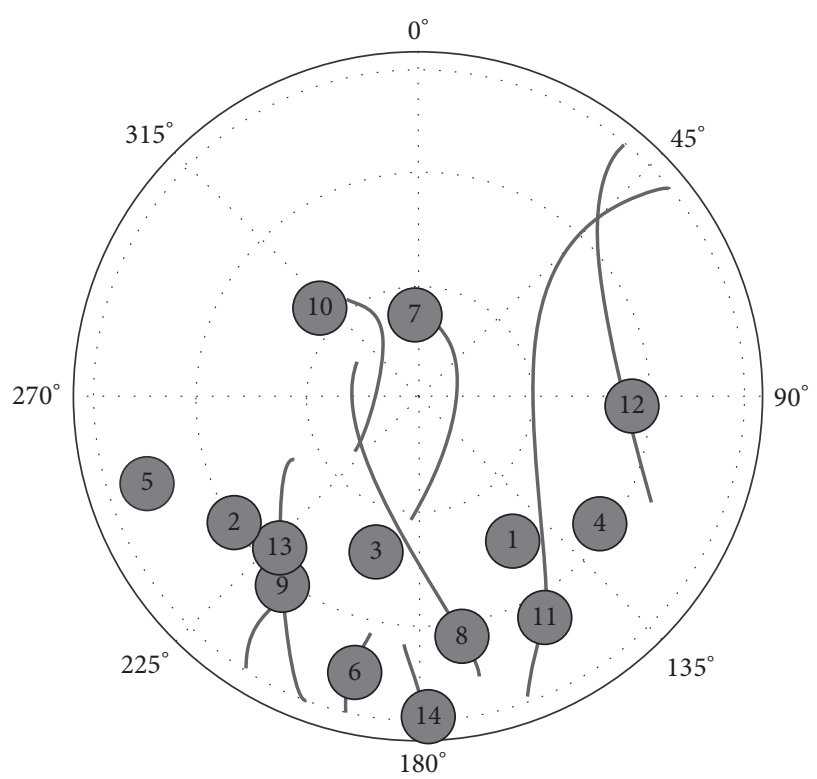

FIgURE 4: Skyplot point of Medium Earth Orbit (MEO) C11 for experiment II (23:50 LT, November 27, 2013).

points, $\mathrm{R} 1$ and $\mathrm{R} 2$ receivers, for monitoring the oil spill. At the R1 observation point, we used the BeiDou IGSO C06 satellite to conduct simulation experiments; this simulation is hereafter referred to as experiment I. Figure 3 shows the Skyplot point of IGSO C06 at experiment I at 08:10 LT on November 27 , 2013. At the R2 observation point, we used the BeiDou MEO C11 satellite to conduct simulation experiments; this simulation is hereafter referred to as experiment II. Figure 4 shows the Skyplot point of MEO C11 for experiment II at 23:50 LT on November 27, 2013. 
TABLE 1: Simulation experiment parameters.

\begin{tabular}{|c|c|c|}
\hline Scenario parameters & Experiment I & Experiment II \\
\hline Transmitter satellite & BeiDou IGSO C06 & BeiDou MEO C11 \\
\hline Time & 08:00-08:10 LT Nov.27, 2013 & 23:40-23:50 LT Nov.27, 2013 \\
\hline Transmitter position $\left(T_{n x}, T_{n y}, T_{n z}\right)$ & $(-1.54,0,3.93) 10^{7} \mathrm{~m}$ & $(-1.45,0,2.38) 10^{7} \mathrm{~m}$ \\
\hline Transmitter velocity $\left(V_{T x}, V_{T y}, V_{T z}\right)$ & $(-1.65,-0.53,-2.03) \mathrm{km} / \mathrm{s}$ & $(-2.21,1.38,-1.58) \mathrm{km} / \mathrm{s}$ \\
\hline Receiver position $\left(R_{n x}, R_{n y}, R_{n z}\right)$ & $(0.0067,0,6.37) 10^{6} \mathrm{~m}$ & $(0.01,0,6.37) 10^{6} \mathrm{~m}$ \\
\hline Receiver velocity $\left(V_{R x}, V_{R y}, V_{R z}\right)$ & $(0,0,0) \mathrm{km} / \mathrm{s}$ & $(0,0,0) \mathrm{km} / \mathrm{s}$ \\
\hline Elevation angle at SP & $31^{\circ}$ & $24^{\circ}$ \\
\hline Oil slick image & \multicolumn{2}{|c|}{ QuickBird multispectral images } \\
\hline Constellations & \multicolumn{2}{|c|}{ BeiDou ephemeris } \\
\hline Coherent integration times & \multicolumn{2}{|c|}{$10 \mathrm{~ms}$} \\
\hline Wind velocity and direction & \multicolumn{2}{|c|}{$6.6 \mathrm{~m} / \mathrm{s}$, along the $x$-axis (ECXI system) } \\
\hline Observation surface, $y$-direction & \multicolumn{2}{|c|}{$[-10-10] \mathrm{km}$} \\
\hline Observation surface, $x$-direction & \multicolumn{2}{|c|}{$[0-10] \mathrm{km}$} \\
\hline Reflect antenna toward & \multicolumn{2}{|c|}{$180^{\circ}$} \\
\hline Receiver height & \multicolumn{2}{|c|}{$15 \mathrm{~m}$} \\
\hline
\end{tabular}

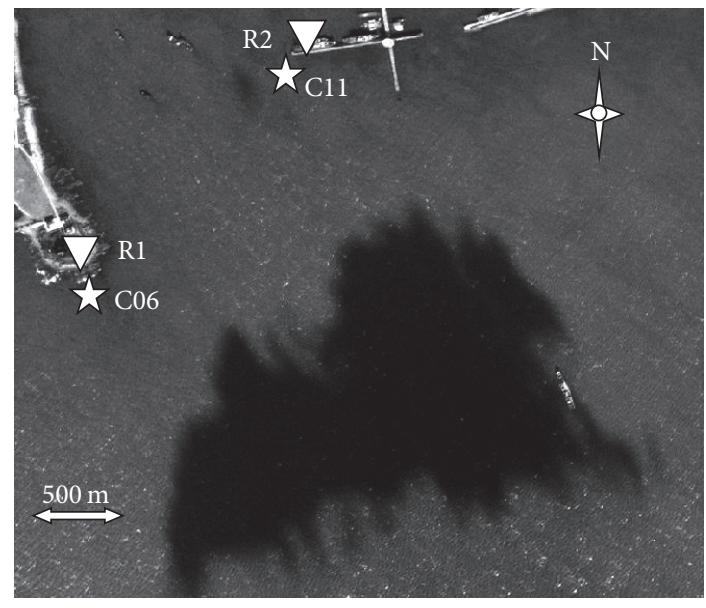

FIGURE 5: QuickBird multispectral images of oil slick at Qingdao on November 27, 2013, and the specular reflection points (SPs) of C06 and $\mathrm{C} 11$.

Figure 5 shows the analog scene monitoring of oil slicks observed on the sea surface, and the black color in the figure represents the shape of the oil slick; the inverted triangles represent the locations of the receiver as R1 and R2; and star points represent the final SPs of C06 (IGSO) and C11 (MEO) in the two experiments. According to the theory of DDM oil slick detection mentioned in Section 2.2, the relative motions of the transmitter and the receiver can produce a Doppler shift to develop DDM for the present of oil slick distinguish in our simulation. The GEO satellite is almost stationary and is therefore not suitable for coastal DDM technology. Thus, the GEO satellite was not considered in our coastal simulation.

In this work, we did not consider the simulation system noise. The influence of the Earth's curvature on the simulation experiment was also not considered because the height of the antenna is only $15 \mathrm{~m}$. On the basis of nearby weather station data from the same period, we determined that the WS observed surface was $6.6 \mathrm{~m} / \mathrm{s}$ and that the distribution was homogeneous over the entire observation surface. Specific descriptions of the simulation scenarios are shown in Table 1. Here, the ECXI system was used [24].

\section{Simulation Results and Discussion}

On the basis of the experiment I simulation parameters, we obtained the averaged scattering coefficients of both oilslicked and clean ocean surfaces over the observation surface by using (6), (8), and (9). Figure 6(a) shows the averaged scattering coefficients of both oil-slicked and clean ocean surfaces in the IGSO scenario. It should be noted that the scattering coefficient of the oil slick decreased more rapidly than that of the clean ocean surface. The figure clearly shows that the scattering coefficients of the two types of surfaces became identical at scattering points $6 \mathrm{~km}$ from the SP, which suggests that the oil slick can be easily detected within a range of about $6 \mathrm{~km}$ from the scattering points in our simulation conditions.

On the basis of experiment II simulation parameters, we obtained the averaged scattering coefficients of both oilslicked and clean ocean surfaces over the observation surface by using (6), (8), and (9). Figure 6(b) shows the averaged scattering coefficients of both oil-slicked and clean ocean surfaces in the MEO scenario. It appears that the averaged scattering coefficients of the two types of surfaces became the same as the scattering points $8 \mathrm{~km}$ from the SP, which suggests that oil slicks can be detected in this range.

As shown in Figure 6, the horizontal axis represents the location of each scattering point relative to the SP over the observation surface, and the vertical axis represents the averaged scattering coefficients of both oil-slicked areas and clean areas. The SP is located at the center of the coordinate system $(x=0, y=0)$.

A contamination mask was derived from the QuickBird multispectral images (Figure 3) for our simulation, which was 


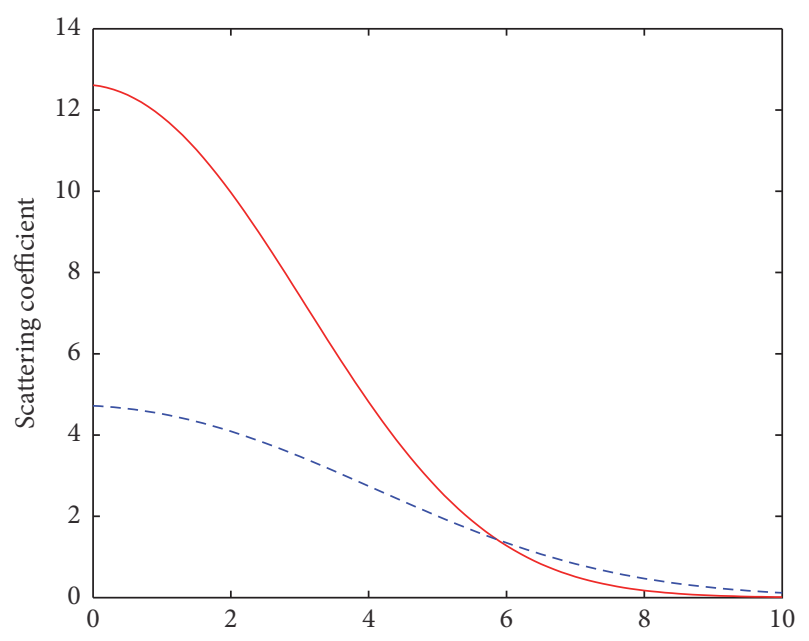

Distance from the points on sea surfaces to the specular point $(\mathrm{km})$

$$
\begin{aligned}
& \text { _- Oil-slicked sea surface } \\
& \text { _- Clean sea surface }
\end{aligned}
$$

(a) Results of the scattering coefficients from the BeiDou Inclined Geosynchronous Orbit (IGSO) simulation experiment

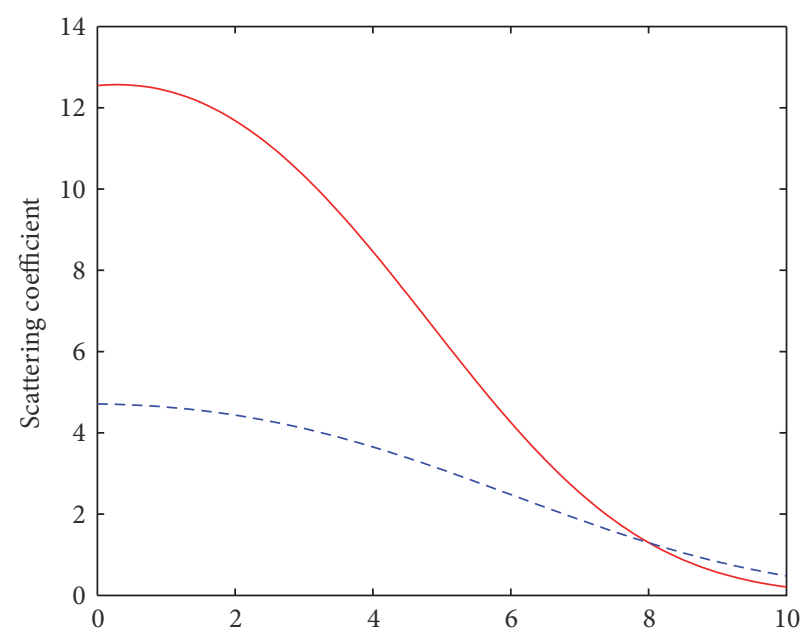

Distance from the points on sea surfaces to the specular point $(\mathrm{km})$

_ Oil-slicked sea surface

- - - Clean sea surface

(b) Results of the scattering coefficients from the BeiDou Medium Earth Orbit (MEO) simulation experiment

FIGURE 6: Scattering coefficients of both oil-slicked areas and clean areas versus distance to the specular reflection point (SP) based on the two different simulation experiments.

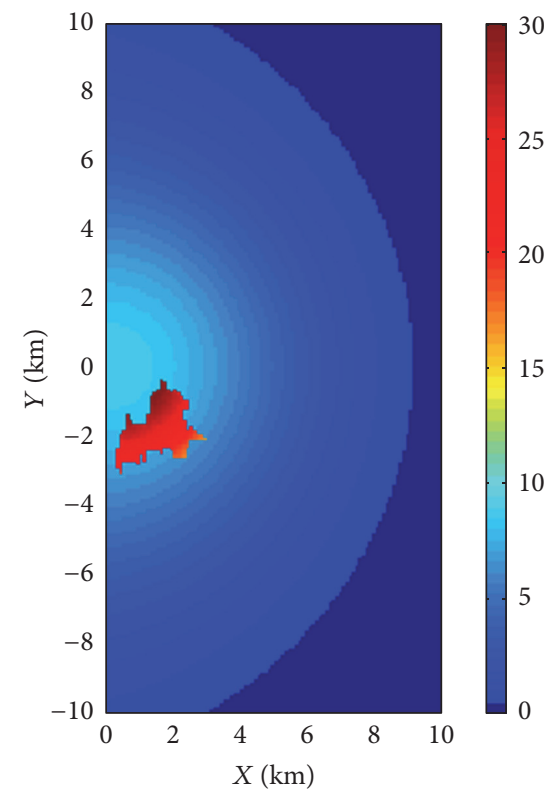

(a) Scattering coefficient distribution based on Inclined Geosynchronous Orbit (IGSO) scenario

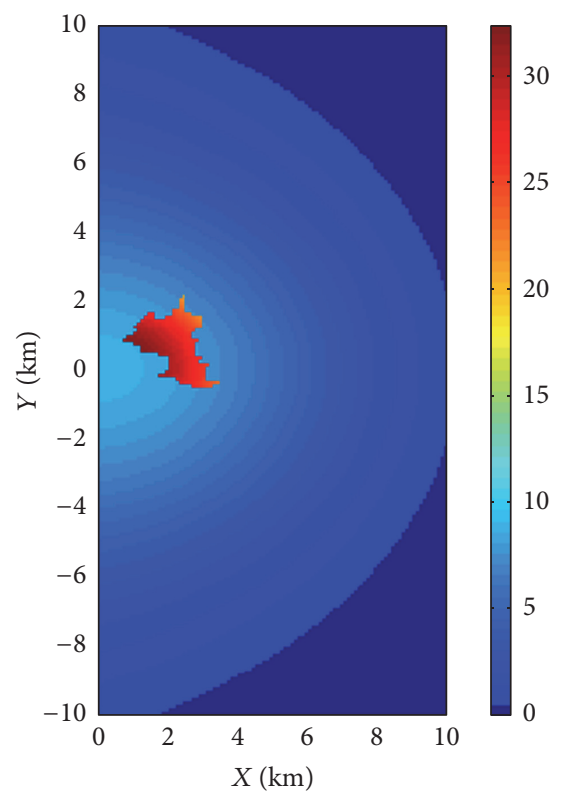

(b) Scattering coefficient distribution based on Medium Earth Orbit (MEO) scenario

FIGURE 7: Simulation results of scattering coefficient distribution from both scenarios.

used as input for the DDM simulation. By combining the design in Figure 6 of the two simulations of oil slick detection scenarios and observed actual oil spill distribution, we obtained the scattering coefficient distributions of each scattering point observation surface including the ocean and the oil slick surface by using (6), (8), and (9). Figures 7(a) and 7(b) show the corresponding scattering coefficient distributions of the two experiments, IGSO and MEO scenarios, respectively. The oil slick area can be clearly distinguished in Figure 7, because it presents a higher scattering coefficient than that of the clean surface. This is attributed to oil damping of the surface waves, which causes more power to be reflected in the 


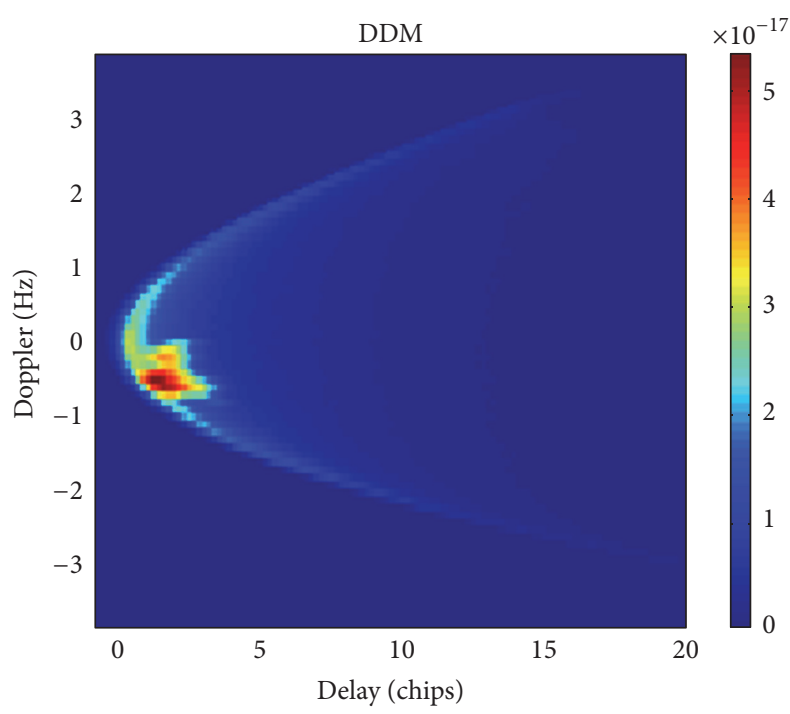

FIgURE 8: Simulated DDM results for simulation experiment I for Inclined Geosynchronous Orbit (IGSO) scenario.

forward direction from this observation surface, although the WS over the entire observation surface is considered uniform.

In Figure 7, the horizontal (vertical) axis indicates the location of each scattering point relative to the SP over the observation surface $x$-direction ( $y$-direction). In the figure, the red (blue) color indicates the scattering coefficient distribution of the oil slick (ocean) surface over the observation surface. The SP is $(x=0, y=0)$.

Both simulation experiments I and II were employed in the DDM based on Figure 7. Figure 8 shows the simulated DDM results for simulation experiment I, which represents the scattering coefficient distribution in the presence of the oil slick shown in Figure 7(a). Figure 9 shows that for experiment II, which represents the scattering coefficient distribution in the presence of the oil slick shown in Figure 7(b). As shown in Figures 8 and 9, the area of the oil spill can be clearly distinguished from the simulated DDMs of the two scenarios, and their ranges are within the theoretical ranges of Figures 6 and 7. Hence, the results of the two simulation experiments indicate that it is feasible to use the BeiDou- $\mathrm{R}$ coastal setup to detect oil slicks over the observed surface. In Figures 8 and 9, the horizontal axis indicates the delay in chips and the vertical axis indicates Doppler. The different colors represent the scattered power values of the corresponding signals.

In the coastal setup, the receiver's location is fixed; thus, the satellite's height and velocity are major factors in the DDM map. A comparison of Figures 8 and 9 revealed that the Doppler shift and the output power from MEO were wider and stronger, respectively, than those from IGSO. This occurred because the velocity of MEO was faster than that of IGSO in the observed period, and the height of MEO was lower than that of IGSO. According to the DDM theory introduced in Section 2.2, if the observed surface points from MEO are more than IGSO, oil slicks can be distinguished more easily from $\mathrm{MEO}$ in our observation period.

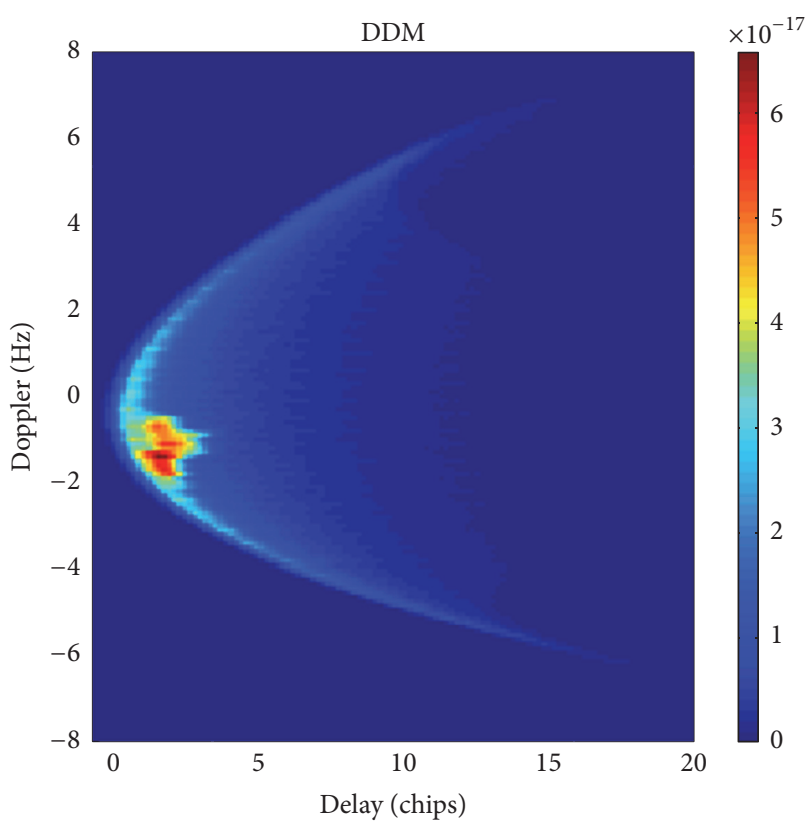

FIGURE 9: Simulated DDM results for simulation experiment II for Medium Earth Orbit (MEO) scenario.

\section{Conclusion}

This study used BeiDou-R simulation to prove the capability of oil slick detection under a coastal scenario based on a DDM obtained by combining the MSS model for oil-slicked/ clean surfaces and the Z-V scattering model. The simulated retrieval process was based on an oil pipeline explosion accident that occurred in 2013 in the district of Qingdao, China. We conducted two simulation experiments of oil slicks by using the BeiDou IGSO C06 and MEO C11 satellites under the same wind conditions. The simulation results showed that the oil-slicked area could be clearly distinguished by using DDM map technology from BeiDou MEO and IGSO satellites with the coastal setup.

By analysis and comparison, the oil slick was detected more easily by MEO, and oil slicks were clearly distinguishable within a coverage area radius of less than $5 \mathrm{~km}$ around the SP in the observation period. Because the receiver's location is fixed in the coastal setup, the satellite's height and velocity affect the DDM results and the oil slick inversion effect. Therefore, a lower satellite height and faster satellite velocity result in stronger output power and better inversion.

\section{Competing Interests}

The authors declare that there is no conflict of interests regarding the publication of this paper.

\section{Acknowledgments}

The work described in this paper was substantially supported by National Natural Science Foundation of China (Grant nos. 41376178, 41401489, and 41506213). 


\section{References}

[1] https://en.wikipedia.org/wiki/Deepwater_Horizon_oil_spill\#cite_ note-area_of_spill-4.

[2] Y. Zhu, X.-M. Qian, Z.-Y. Liu, P. Huang, and M.-Q. Yuan, "Analysis and assessment of the Qingdao crude oil vapor explosion accident: lessons learnt," Journal of Loss Prevention in the Process Industries, vol. 33, pp. 289-303, 2015.

[3] C. S. L. Grimaldi, D. Casciello, I. Coviello, T. Lacava, N. Pergola, and V. Tramutoli, "Satellite oil spill detection and monitoring in the optical range," in Proceedings of the 30th IEEE International Geoscience and Remote Sensing Symposium (IGARSS '10), July 2010.

[4] A. H. S. Solberg, C. Brekke, and P. O. Husøy, "Oil spill detection in Radarsat and Envisat SAR images," IEEE Transactions on Geoscience and Remote Sensing, vol. 45, no. 3, pp. 746-754, 2007.

[5] S. Gleason, S. Lowe, and V. Zavorotny, "Remote sensing using bistatic GNSS reflections," in GNSS Applications and Methods, S. Gleason and D. Gebre-Egziabher, Eds., Artech House, Norwood, Mass, USA, 2009.

[6] M. Martin-Neira, "A passive reflectometry and interferometry system (PARIS): application to ocean altimetry," ESA Journal, vol. 17, no. 4, pp. 331-355, 1993.

[7] A. Rius, E. Cardellach, and M. Martín-Neira, "Altimetric analysis of the sea-surface GPS-reflected signals," IEEE Transactions on Geoscience and Remote Sensing, vol. 48, no. 4, pp. 2119-2127, 2010.

[8] H. Park, E. Valencia, A. Camps, A. Rius, S. Ribó, and M. MartínNeira, "Delay tracking in spaceborne GNSS-R ocean altimetry," IEEE Geoscience and Remote Sensing Letters, vol. 10, no. 1, pp. 57-61, 2013.

[9] Y. Zhang, L. Tian, W. Meng, Q. Gu, Y. Han, and Z. Hong, "Feasibility of code-level altimetry using coastal BeiDou reflection (BeiDou-R) setups," IEEE Journal of Selected Topics in Applied Earth Observations and Remote Sensing, vol. 8, no. 8, pp. 41304140, 2015.

[10] A. Ghavidel, D. Schiavulli, and A. Camps, "Numerical computation of the electromagnetic bias in GNSS-R altimetry," IEEE Transactions on Geoscience and Remote Sensing, vol. 54, no. 1, pp. 489-498, 2016.

[11] J. L. Garrison, A. Komjathy, V. U. Zavorotny, and S. J. Katzberg, "Wind speed measurement using forward scattered GPS signals," IEEE Transactions on Geoscience and Remote Sensing, vol. 40, no. 1, pp. 50-65, 2002.

[12] N. Rodriguez-Alvarez, D. M. Akos, V. U. Zavorotny, J. A. Smith, A. Camps, and C. W. Fairall, "Airborne GNSS-R wind retrievals using delay-doppler maps," IEEE Transactions on Geoscience and Remote Sensing, vol. 51, no. 1, pp. 626-641, 2013.

[13] W. Li, F. Fabra, D. Yang et al., "Initial results of typhoon wind speed observation using coastal GNSS-R of BeiDou GEO satellite," IEEE Journal of Selected Topics in Applied Earth Observations and Remote Sensing, vol. 9, no. 10, pp. 1-10, 2016.

[14] F. Soulat, M. Caparrini, O. Germain, P. Lopez-Dekker, M. Taani, and G. Ruffini, "Sea state monitoring using coastal GNSS-R," Geophysical Research Letters, vol. 31, no. 21, 2004.

[15] M. Caparrini, A. Egido, F. Soulat et al., "Oceanpal ${ }^{\circledR}$ : monitoring sea state with a GNSS-R coastal instrument," in Proceedings of the IEEE International Geoscience and Remote Sensing Symposium (IGARSS '07), pp. 5080-5083, Barcelona, Spain, June 2007.

[16] K. M. Larson, R. D. Ray, F. G. Nievinski, and J. T. Freymueller, "The accidental tide gauge: a GPS reflection case study from
Kachemak Bay, Alaska," IEEE Geoscience and Remote Sensing Letters, vol. 10, no. 5, pp. 1200-1204, 2013.

[17] M. B. Rivas, J. A. Maslanik, and P. Axelrad, "Bistatic scattering of GPS signals off arctic sea ice," IEEE Transactions on Geoscience and Remote Sensing, vol. 48, no. 3, pp. 1548-1553, 2010.

[18] F. Fabra, E. Cardellach, A. Rius et al., "Phase altimetry with dual polarization GNSS-R over sea ice," IEEE Transactions on Geoscience and Remote Sensing, vol. 50, no. 6, pp. 2112-2121, 2012.

[19] Z. Yun, M. Wanting, G. Qiming et al., "Detection of bohai bay sea ice using GPS-reflected signals," IEEE Journal of Selected Topics in Applied Earth Observations and Remote Sensing, vol. 8, no. 1, pp. 39-46, 2015.

[20] E. Valencia, A. Camps, H. Park et al., "Oil slicks detection using GNSS-R," in Proceedings of the IEEE International on Geoscience and Remote Sensing Symposium (IGARSS '11), vol. 142, pp. 43834386, July 2011.

[21] E. Valencia, A. Camps, N. Rodriguez-Alvarez, H. Park, and I. Ramos-Perez, "Using GNSS-R imaging of the ocean surface for oil slick detection," IEEE Journal of Selected Topics in Applied Earth Observations and Remote Sensing, vol. 6, no. 1, pp. 217223, 2013.

[22] C. Li and W. Huang, "Sea surface oil slick detection from GNSSR delay-Doppler maps using the spatial integration approach," in Proceedings of the IEEE Radar Conference (RadarCon '13), pp. 1-6, Ottawa, Canada, May 2013.

[23] C. Li, W. Huang, and S. Gleason, "Dual antenna space-based GNSS-R ocean surface mapping: oil slick and tropical cyclone sensing," IEEE Journal of Selected Topics in Applied Earth Observations and Remote Sensing, vol. 8, no. 1, pp. 425-435, 2015.

[24] C. Li and W. Huang, "Simulating GNSS-R delay-doppler map of oil slicked sea surfaces under general scenarios," Progress in Electromagnetics Research B, vol. 48, pp. 61-76, 2013.

[25] M. Z. Bhuiyan, S. Söderholm, S. Thombre, L. Ruotsalainen, and $\mathrm{H}$. Kuusniemi, "Overcoming the challenges of beidou receiver implementation," Sensors, vol. 14, no. 11, pp. 22082-22098, 2014.

[26] V. U. Zavorotny and A. G. Voronovich, "Scattering of GPS signals from the ocean with wind remote sensing application," IEEE Transactions on Geoscience and Remote Sensing, vol. 38, no. 2, pp. 951-964, 2000.

[27] J. F. Marchan-Hernandez, A. Camps, N. Rodriguez-Alvarez, E. Valencia, X. Bosch-Lluis, and I. Ramos-Perez, "An efficient algorithm to the simulation of delay-doppler maps of reflected global navigation satellite system signals," IEEE Transactions on Geoscience and Remote Sensing, vol. 47, no. 8, pp. 2733-2740, 2009.

[28] S. Gleason, "Towards sea ice remote sensing with space detected GPS signals: demonstration of technical feasibility and initial consistency check using low resolution sea ice information," Remote Sensing, vol. 2, no. 8, pp. 2017-2039, 2010.

[29] F. Soulat, Sea surface remote sensing with GNSS and sunlight reflections [Ph.D. thesis], Universitat Politècnica de Catalunya, Catalunya, Spain, 2003.

[30] C. Cox and W. Munk, "Measurement of the roughness of the sea surface from photographs of the Sun's glitter," Journal of the Optical Society of America, vol. 44, no. 11, pp. 838-850, 1954.

[31] S. J. Katzberg, O. Torres, and G. Ganoe, "Calibration of reflected GPS for tropical storm wind speed retrievals," Geophysical Research Letters, vol. 33, no. 18, Article ID L18602, 2006. 


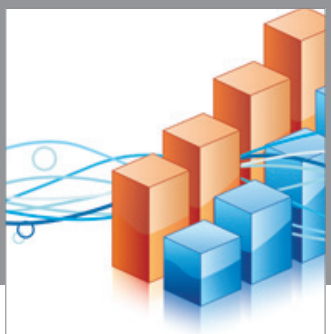

Advances in

Operations Research

vatem alat4

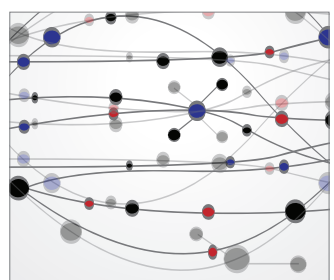

\section{The Scientific} World Journal
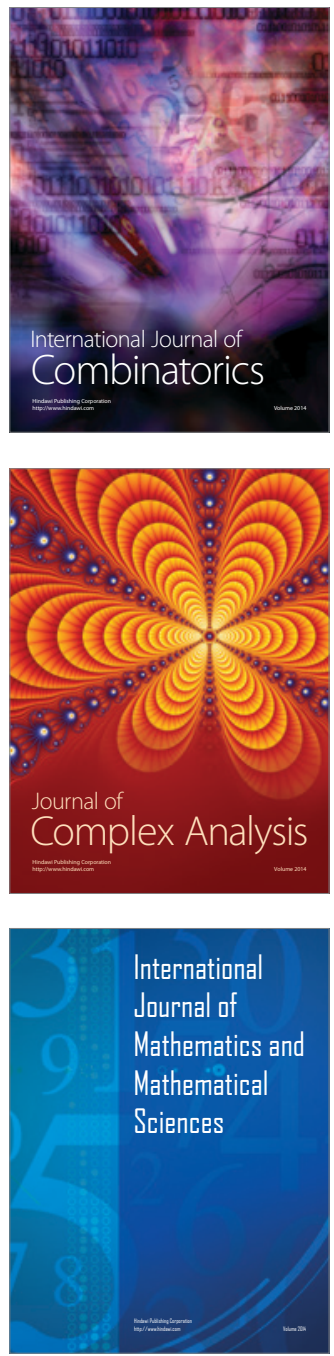
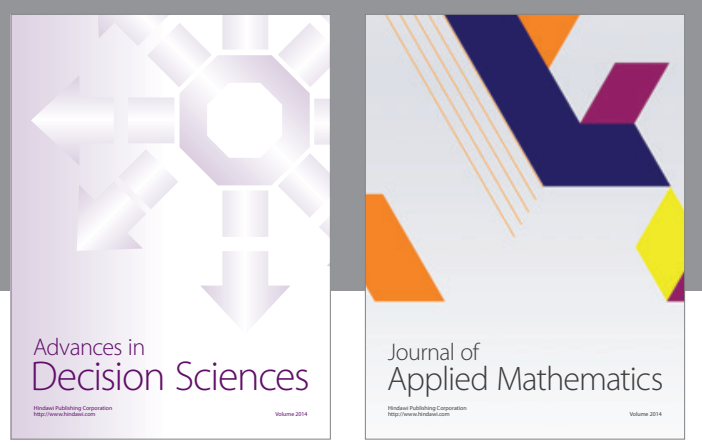

Algebra

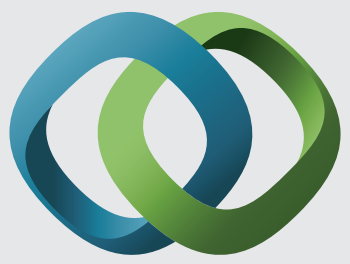

\section{Hindawi}

Submit your manuscripts at

https://www.hindawi.com
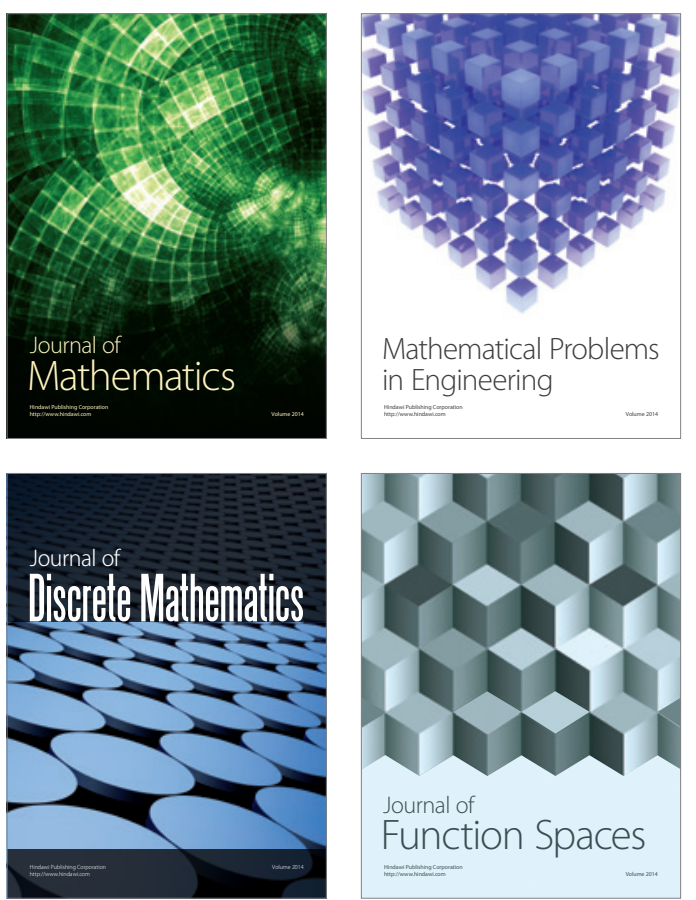

Mathematical Problems in Engineering
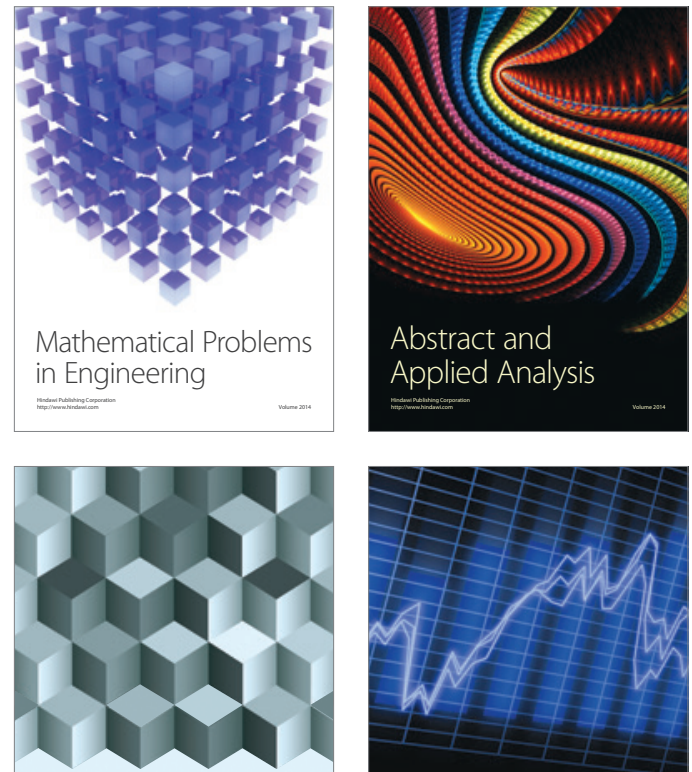

Journal of

Function Spaces

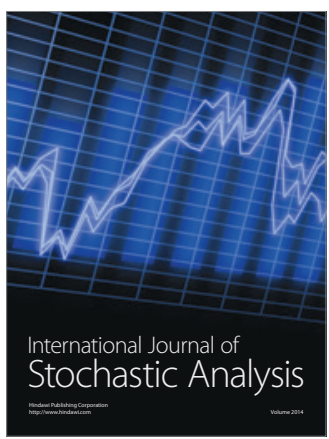

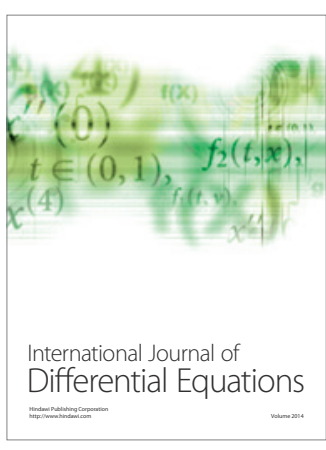
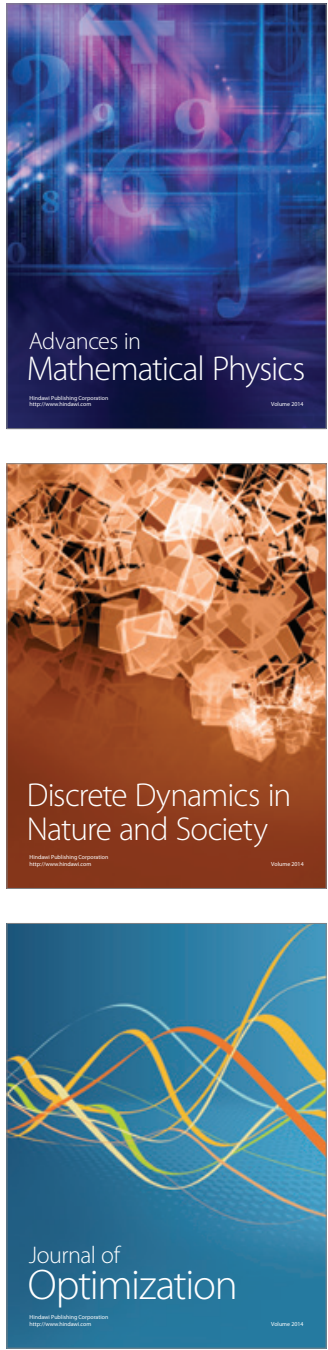